\title{
e-Phaïstos
}

e-Phaïstos

Revue d'histoire des techniques / Journal of the history

of technology

VII-1 | 2019

Le travail humain

\section{Histoire et techniques de cultures du vivrier en \\ Côte d'Ivoire, de la transformation à la commercialisation : le cas du manioc (1960-2000)}

History of Subsistence Farming and its technology, from Processing to

Marketing : The Cassava's Case, 1960-2000

\section{Daouda Diarrassouba}

\section{OpenEdition}

Journals

Édition électronique

URL : https://journals.openedition.org/ephaistos/4174

DOI : 10.4000/ephaistos.4174

ISSN : 2552-0741

Éditeur

IHMC - Institut d'histoire moderne et contemporaine (UMR 8066)

Référence électronique

Daouda Diarrassouba, « Histoire et techniques de cultures du vivrier en Côte d'Ivoire, de la

transformation à la commercialisation : le cas du manioc (1960-2000) », e-Phaïstos [En ligne], VII-1 |

2019, mis en ligne le 06 avril 2019, consulté le 21 septembre 2021. URL : http://

journals.openedition.org/ephaistos/4174; DOI : https://doi.org/10.4000/ephaistos.4174

Ce document a été généré automatiquement le 21 septembre 2021.

Tous droits réservés 


\section{Histoire et techniques de cultures du vivrier en Côte d'Ivoire, de la transformation à la commercialisation : le cas du manioc (1960-2000)}

History of Subsistence Farming and its technology, from Processing to Marketing : The Cassava's Case, 1960-2000

\section{Daouda Diarrassouba}

Thèse : références bibliographiques

Daouda DIARROUSSABA, Histoire et techniques de cultures du vivrier en Côte d'Ivoire, de la transformation à la commercialisation : le cas du manioc (1960-2000), Thèse de doctorat en Histoire économique et des techniques, Université Alassane Ouattara, Bouaké (Côte d'Ivoire), soutenue le 22 janvier 2018, un volume de texte (430 p).

Directeur de thèse

Gaston Ouakoubo GNABRO, Maître de conférence, Université Péléforo Gon Coulibaly de Korhogo

Jury :

Anne-Françoise GARÇON, Professeur émérite en histoire des techniques, Université Paris 1 Panthéon-Sorbonne (Présidente)

Gaston Ouakoubo GNABRO, Maître de conférence, Université Péléforo Gon Coulibaly de Korhogo

Antoine Koffi GOLE, Maître de conférence, Université Alassane Ouattara de Bouaké 
Raphaël Békoin TANOH, Maître de conférence, Université Alassane Ouattara de Bouaké

1 Votre Éminence, Madame la Présidente du jury, Honorables messieurs les membres du Jury, Très chers maîtres,

2 C'est avec un réel plaisir et une joie singulière que nous prenons la parole. Et, comprenez notre émotion! Avoir droit à la parole en pareil circonstance, devant des sommités du monde scientifique que vous représentez, est pour l'étudiant que nous sommes, un grand honneur. Merci donc madame la présidente de nous avoir donné la parole.

3 Madame la Présidente du jury, honorables messieurs les membres du Jury, très chers maitres, permettez-nous avant tout propos, de vous saluer et de vous exprimer toute notre gratitude pour l'intérêt que vous avez bien voulu porter à notre travail de recherche, en prenant part à ce jury et en nous permettant de soutenir cette thèse unique de Doctorat. Madame la présidente, Professeur émérite Anne-François GARÇON, nous tenons à vous adresser toute notre reconnaissance pour les remarques que vous avez apportées à notre travail. Comme une bonne mère, vous aviez accepté de faire un long voyage pour venir présider ce jury, malgré toutes les contraintes professionnelles. Nous vous disons merci. Nous vous en sommes très reconnaissants.

4 Permettez-nous madame la présidente de témoigner notre gratitude à un Homme. Cet Homme a été, et restera tout pour nous à vie. Il est pour nous à la fois un père (par son affection vraie de parent et sa bonté), un aîné (par sa simplicité et sa gentillesse) et un maitre (par sa rigueur dans le travail et son grand désir de transmettre son savoir). Nous avons nommé, le Professeur GNABRO Ouakoubo Gaston. Merci du fond du cœur Professeur, pour tout ce que vous avez fait et continuer de faire pour nous. Votre passion pour la recherche a été communicative, et grâce à votre rigueur dans le travail, votre humanisme, votre bienveillance et grâce à la confiance puis à l'autonomie que vous nous avez concédé, nous nous sommes véritablement épanouis au fil de ces quelques années de recherches. Grâce à vous, nous sommes aujourd'hui à cette instance solennelle de soutenance de notre thèse. Nous vous en exprimons toute notre reconnaissance et notre plus profond respect très cher maître.

5 Nos remerciements vont au maître, le professeur GOLE Koffi Antoine. Cher maître, vos travaux de recherche et différentes orientations, il faut le dire, ont été pour ce travail, ce que les yeux représentent pour le corps. Nous nous inclinons devant votre éminence et vous disons infiniment merci, grand maître. Permettez-moi, madame la présidente de tourner maintenant le regard vers un homme de science, le Professeur TANOH Raphael Békoin. Très cher maître, infiniment merci d'avoir accepté d'être là, à cette soutenance. Votre présence est un véritable honneur pour nous. Merci pour votre promptitude avec laquelle vous avez répondu présent. Nous voudrions enfin saluer et dire merci à tous les enseignants du département d'histoire de l'université Alassane Ouattara et à nos condisciples et à nos parents ici présents.

6 Après cette phase protocolaire, revenons madame la présidente, à l'intérêt de la recherche, portant sur l'histoire et les techniques de cultures du vivrier en Côte d'Ivoire, de la transformation à la commercialisation, le cas du manioc de 1960 à 2000. 


\section{Pourquoi le choix de ce sujet?}

7 Les africains de façon générale et en particulier les ivoiriens, avant l'arrivée des européens avaient une agriculture basée sur les cultures vivrières. En fait, les Ivoiriens se consacraient à l'agriculture de subsistance destinée à la consommation directe. L'agriculture était alors tournée vers l'autoconsommation des cellules familiales ivoiriennes. C'est le surplus de la production, qui était destinée à la commercialisation. C'est pourquoi, les cultures vivrières introduites par les européens ont été facilement acceptées par les ivoiriens.

8 L'introduction des cultures de rente par les Européens et leur intensification en Côte d'Ivoire vont bouleverser le système cultural des ivoiriens. Les cultures vivrières de façon générale et en particulier le manioc ont été délaissés au profit des cultures industrielles destinées à ravitailler les industries de la métropole. Cependant, il faut souligner que les cultures d'exportation n'ont pas été acceptées automatiquement par les ivoiriens. En effet, C'est la richesse créée par les cultures de rente qui a amené les Ivoiriens à accepter avec le temps les cultures de rente. En clair, elles avaient participé à l'émergence d'une bourgeoisie locale dans la colonie ivoirienne pendant la colonisation et après la colonisation. Le colonisateur n'avait pas fait la promotion du manioc auprès des populations ivoiriennes comme les autres cultures vivrières, à savoir, l'igname et le maïs. Le manioc fut délaissé car aux yeux des colons, il n'était pas une culture d'avenir pour la colonie ivoirienne. Chez les peuples de la savane, le manioc était considéré comme une culture secondaire. Cependant, le manioc, après l'indépendance commence à avoir de l'importance dans toutes les zones de Côte d'Ivoire. Il a même tendance à supplanter les cultures industrielles dans le pays. De tous les féculents traditionnels, le manioc est certainement celui dont l'expansion est la plus agressive, et en même temps la plus souhaitable. ${ }^{1} \mathrm{Au}$ fil du temps, il devient de plus en plus indispensable dans la vie quotidienne des ivoiriens. Cet intérêt accordé subitement au manioc a constitué pour nous, une raison pour le choix de notre sujet. Cet aspect constitue la raison subjective de notre travail.

9 Aussi, faut-il noter que plusieurs travaux ont été menées sur le manioc dans plusieurs disciplines par des chercheurs étrangers et des ivoiriens ${ }^{2}$. Ces études n'ont pas été menées de façon spécifique en histoire. Même si elles existent c'est de manière générale sur les cultures vivrières de la Côte d'Ivoire.

10 Le choix du sujet « Histoire et Techniques des cultures du vivrier en Côte d'Ivoire, de la transformation à la commercialisation: le cas du manioc de 1960 à 2000 ", n'est pas fortuit pour l'écriture de cette Thèse Unique de Doctorat. Ce thème permettra de lever le voile sur la culture du manioc, en montrant comment le manioc ${ }^{3}$, qui était une culture marginalisée est devenu indispensable dans la vie quotidienne des ivoiriens. A travers cette étude, nous apportons une contribution à la recherche en histoire sur le manioc. Cette thèse permettra de faire un tableau synoptique sur le manioc en Côte d'Ivoire, en partant des techniques de culture, de transformation à sa commercialisation. Ces différents aspects sont très importants dans l'élaboration de cette thèse. Tel est le volet objectif de notre travail.

11 Tous ces aspects soulignés, nous ont amené à choisir ce sujet pour avoir une connaissance sur la culture du manioc en Côte d'Ivoire. Pour nous, les origines du manioc doivent être connue par les ivoiriens et les autres africains. Mais, au-delà, le manioc a une portée et un intérêt pour les populations mondiales. Il fait l'objet de 
convoitise sur tous les continents du monde. Il est très utilisé en Europe pour l'alimentation des animaux. Sur le continent asiatique, dans les États du Pacifique et Caraïbes, il est utilisé pour la consommation humaine et pour la consommation animale. L'Afrique est le premier producteur de cette denrée. La Côte d'Ivoire, depuis des décennies est dans le peloton des grands producteurs du monde. Le manioc a tellement de l'importance, qu'au fil du temps, on peut même voir des européens et même des asiatiques vendre des produits faits à base du manioc. En France, des chinois sont vendeurs de l'attiéké et d'autres produits d'origine africaine à base du manioc. Il est donc important de montrer la place de cette culture en Côte d'Ivoire qui est la deuxième culture vivrière après l'igname.

\section{Quel est l'objectif de ce travail ?}

L'objectif de notre travail consiste à montrer comment manioc, une culture marginalisée devient indispensable dans la vie des Ivoiriens. Cette utilité se justifie tant au niveau alimentaire qu'au niveau pécuniaire. Trois objectifs spécifiques se dégagent :

- Le premier s'inscrit dans un contexte historique parce que la culture du manioc a traversé plusieurs siècles. Il permettra de connaitre les différentes techniques culturales en Côte d'Ivoire.

- Le second objectif met en exergue les différentes techniques de conservation et de transformation dans le monde et en particulier en Côte d'Ivoire. Il permettra de connaitre la diffusion du savoir-faire des différents peuples concernant ces différentes techniques.

- Le troisième objectif de notre travail aborde la commercialisation du manioc et ses dérivés en passant par l'évolution de la production mondiale et nationale ivoirienne.

Ces objectifs sont très importants, ils permettent de cadrer le travail dans son ensemble. Ils permettent de comprendre mieux le sujet de la recherche et à l'orienter $\mathrm{du}$ point de vue de la méthodologie. Ils aident à décliner les différents aspects du travail.

\section{Comment avons-nous procédé pour réaliser ce travail (la méthodologie)?}

Pour la réalisation de ce travail, nous avons interrogé des sources et des ouvrages. S'agissant des sources nous avons interrogé les sources d'archives de la Côte d'Ivoire, les sources imprimées et des personnes ressources par l'enquête orale. Pour ce qui est des ouvrages, nous avons lu des ouvrages généraux, des ouvrages spécifiques sur le manioc, des thèses, des mémoires et des articles.

Les informations recueillies dans les documents, ont été classées et confrontées entre elles. Il en était de même pour celles tirées des sources orales. Avant de valider les informations, nous les avons soumis à la critique historique. C'est à l'issue de ce procédé que nous avons validé les informations qui semblent faire l'unanimité. On a donc essayé de confronter et de comparer les différentes sources entre elles et avec la bibliographie grâce au système de recoupement de l'information. Après cela, nous avons poursuivi la recherche sur les informations contradictoires, afin de déceler la vérité. En définitive, nous avons approché, avec mesure, les sources, le tout étant de ne 
pas perdre de vue les exigences de l'authenticité et la véracité des faits. Nous avons, à partir des données recueillies et traitées, structuré le travail autour de trois grandes parties. Chaque partie est composée de trois chapitres qui permettent le bon déroulement du travail. Tous les chapitres sont composés de sous chapitres.

La première partie de notre travail met l'accent sur l'histoire et les techniques de culture du manioc en Côte d'Ivoire des années 1960 aux années 1980. Le premier chapitre permet de connaitre les origines du manioc dans le monde et en Côte d'Ivoire. En effet, nous abordons de façon poussée, les origines de la culture du manioc. Ensuite, nous mettons l'accent sur les différents caractères du manioc à travers le monde. Puis, nous montrons la composition du manioc et les différentes variétés de manioc cultivées en Côte d'Ivoire. Le deuxième chapitre nous permet de connaitre les conditions de développement de la culture du manioc en Côte d'ivoire. En clair, ce chapitre se focalise sur les différents modes d'accès à la terre et les différents types de main-d'œuvre des exploitations agricoles. Quant au dernier chapitre de cette première partie, il lève le voile sur les techniques de culture du manioc en Côte d'Ivoire. Dans ce chapitre, nous aborderons d'abord, les différentes étapes pour la création d'une plantation de manioc. Ensuite, nous montrons les différentes techniques de protection des cultures contre les maladies et les ennemis. Et enfin, nous étudions le premier centre mécanisé de la culture du manioc en Côte d'Ivoire qui est le centre de Toumodi en 1977.

Intitulée " de la conservation à la transformation du manioc : 1983-1990 ", la deuxième partie nous permet de connaitre les techniques de conservation et de transformation du manioc. Le premier chapitre aborde l'évolution des techniques de conservation des racines tubéreuses du manioc depuis sa domestication en Amérique du Sud. Puis nous étudions les différentes techniques traditionnelles de conservation du manioc en Côte d'Ivoire, en analysant les techniques traditionnelles améliorées de conservation des racines tubéreuses. Le dernier aspect de ce travail aborde les procédés de conservation semi-industriels et moderne du manioc à l'état frais. Le deuxième chapitre se focalise sur les différentes transformations du manioc. Primo, nous analysons les différentes transformations traditionnelles du manioc en Côte d'Ivoire. Secundo, nous mettons l'accent sur les différents procédés et technologies semi-industriels de transformation $\mathrm{du}$ manioc. Tercio, nous analysons les différentes transformations industrielles du manioc. Enfin, le dernier chapitre de cette partie aborde les différentes transformations du manioc dans le monde. En clair, le premier sous-chapitre présente les différentes transformations du manioc en Afrique Centrale. Dans cette zone le manioc fait l'objet d'une grande consommation, en effet. Le deuxième chapitre analyse les différentes transformations en Amérique Latine et en Asie. Le dernier sous-chapitre met l'accent sur la fabrication des matériels améliorés de transformation du manioc, ainsi que sur la vente de ces matériels.

La troisième et dernière partie de notre travail, est intitulée «la commercialisation $d u$ manioc et ses dérivés en Côte d'Ivoire: 1990-2000». Cette dernière partie interroge le marché mondial du manioc et le marché intérieur de la Côte d'Ivoire. Dans le premier chapitre, nous analysons dans un premier temps, la situation du manioc dans le monde, en passant en revue les différentes utilisations du manioc. Ensuite, nous abordons l'organisation du marché mondial du manioc et ses dérivés. Enfin, nous analysons la place qu'occupe la Côte d'Ivoire dans ce marché international. Le deuxième chapitre de notre travail étudie le commerce interne du manioc en Côte d'Ivoire, en étudiant les facteurs de l'essor de la commercialisation du manioc et ses dérivés, les acteurs du 
commerce de manioc et dérivés en Côte d'Ivoire, la place du manioc au niveau des frontières Est du pays. Enfin, le dernier chapitre se focalise sur l'impact de la culture du manioc sur les populations ivoiriennes. Dans ce chapitre, nous montrons d'abord, le circuit d'approvisionnement de la ville d'Abidjan en produit de manioc. Ensuite, nous abordons les effets induits du manioc sur la population ivoirienne, en particulier les sources supplémentaires de revenus qu'il apporte.

\section{Que retenir de ce travail ?} certaines régions de la Côte d'Ivoire. Dans le sud, il est consommé sous la forme la plus connue appelée « attiéké » qui est un met ressemblant beaucoup au couscous. Au nord, il est consommé sous forme de farine cuite appelée «tôh » et à l'ouest sous forme de pâte communément appelée "foutou». Au centre-ouest, le manioc est utilisé pour préparer un repas dénommé «placali» qui est un aliment beaucoup prisé par les natifs de cette région. Placali est une pâte confectionnée ou produite à partir de la farine de manioc que l'on malaxe plusieurs minutes, au moyen d'une spatule, sur un feu doux, pendant la cuisson. Bien que le manioc représente un aliment de substitution pour les peuples qui le consomment occasionnellement, c'est l'une des denrées alimentaires que l'on retrouve dans tous les ménages à travers la consommation de l'attiéké. Il y a presque deux décennies que la demande de l'attiéké sur le marché ivoirien et sur celui des pays limitrophes du nord de la Côte d'Ivoire augmente chaque année. Dans chaque ville de la Côte d'Ivoire, l'on trouve des petits restaurants de fortune appelés dans le jargon ivoirien "garbadrome» dans lesquels l'attiéké est vendu et consommé en grande quantité. Cette consommation importante engendre une hausse de la demande. Pour satisfaire cette demande croissante, certains cultivateurs de ce tubercule ont augmenté de façon considérable leur superficie cultivable et de nouveaux cultivateurs se sont intéressés à l'activité ; rejoignant ainsi le nombre de producteurs. C'est alors que la production de manioc s'est étendue sur une grande partie du territoire national et est devenue une activité économique génératrice de revenu pour certains paysans.

Le processus de création d'une exploitation de manioc est le même partout en Côte d'Ivoire. Cependant, des différences existaient au niveau du planting des boutures et de l'entretien. Par exemple chez les Ehotilé, les boutures sont mises en terre de manière verticale ce qui n'est pas le cas chez les Ebrié. Au niveau de l'entretien, c'est la zone d'Abidjan qui utilise de l'engrais, par contre l'engrais n'est pas utilisé dans les autres zones de la Côte d'Ivoire. La culture du manioc devient importante aux yeux des autorités ivoiriennes à partir de 1977. L'importance du manioc se manifesta par la mise en place d'un centre de culture et de transformation. Ce projet fut implanté dans la ville de Toumodi. Il fut un projet de grande envergure, totalement mécanisé, de la culture à la transformation du manioc. Mais, le projet rencontra très tôt des difficultés qui ont entrainé sa disparition.

Néanmoins, la culture du manioc devient plus importante à partir des années 1980, en raison de l'intérêt accordé par l'état ivoirien aux cultures vivrières en dehors du riz. Les techniques de conservation et de transformation ont été améliorées au fil du temps par les chercheurs du monde entier et en particulier par les chercheurs ivoiriens. La conservation des racines tubéreuses de manioc sur pied domine en effet en Côte d'Ivoire. Mais cette technique, la plus ancienne des techniques de conservation,

e-Phaïstos, VII-1 | 2019 
constitue une perte en surface cultivable pour les exploitants. Et la conservation des racines tubéreuses est limitée après récolte. L'amélioration des procédés de conservation eut donc pour but de commercialiser le manioc sur une longue période ou sur de longues distances.En outre, les techniques de transformation traditionnelles ont été améliorées avec l'introduction de nouveaux éléments ou à travers la mécanisation des chaînes de production des dérivés de manioc. Le manioc passa des techniques de transformation autochtones aux techniques de transformation semi-industrielles pour atteindre le système purement industriel. L'amélioration des techniques est effective en Côte d'Ivoire. Faute de moyens, les africains plus précisément les ivoiriens ont mis en place des machines améliorées, accessibles à tous.

Le troisième et le dernier axe de notre étude s'est consacré à la commercialisation du manioc et ses dérivés dans le monde et en particulier en Côte d'Ivoire. Le commerce mondial est lié aux différentes demandes du manioc et dérivés des pays européens et de quelques pays asiatiques comme la Chine. Il faut savoir que les grands pays exportateurs du manioc sont les pays asiatiques tels que la Thaillande, l'Indonésie. Les pays africains constituent les grands producteurs de manioc. Mais, leur production est consacrée à la consommation nationale. La Côte d'Ivoire occupe une place négligeable dans le commerce international du manioc et dérivés. La quantité exportée par le pays est très faible. Le commerce ivoirien est géré par des particuliers. Ce qui fait que les quantités exportées ne sont pas connues avec exactitude.

Néanmoins, les cultures vivrières ont bénéficié très tôt d'une politique de commercialisation engagée par l'État ivoirien avec la mise en place de plusieurs structures dont la dernière est l'OCPV, chargée de faciliter la commercialisation du vivrier. A cela, s'ajouta l'urbanisation rapide au lendemain de l'indépendance ivoirienne. C'est dans ce contexte que le manioc gagne en notoriété dans les villes ivoiriennes grâce à ces produits dérivés comme l'attiéké et le placali. Le manioc gagne encore du terrain avec la crise économique des années 1980. La crise a occasionné une baisse du pouvoir d'achat des ivoiriens plus précisément des ménages ivoiriens. Le manioc gagne enfin du terrain par le biais d'une filière très bien organisée par les femmes ivoiriennes. Dans le domaine du commerce interne, les femmes se sont organisées en coopérative pour la vente de leurs produits dont le manioc. Le circuit est formé de producteurs ou productrices, de grossistes et de détaillantes, et il possède un réseau international qui diffuse désormais ses produits en France et dans le reste de l'Europe. Le manioc a eu une influence considérable sur la vie des ivoiriens, les femmes en particulier, en leur permettant de se prendre en charge dans les zones rurales et même en zones urbaines.

Madame la présidente du jury, messieurs les membres du jury, cela a été un honneur et un plaisir pour nous de vous avoir fait partager les conclusions de cette thèse de Doctorat. Nous nous réjouissons par avance du dialogue qui suivra notre exposé et qui permettra sans nul doute d'approfondir, ensemble, les interrogations et les questionnements qui ont animé ce travail. Nous vous remercions très chers maîtres de votre aimable attention. Comme nous tenons également à remercier les personnes ici présentes dans la salle pour leur participation. 


\section{NOTES}

1. CAMARA Camille, "les cultures vivrières en République de Côte d'Ivoire", Annales de géographie, 1984, p. 442.

2. Ces études ont été menées par des géographes et des agronomes.

3. Manihot Esculenta Crantz : c'est le nom scientifique du manioc.

\section{RÉSUMÉS}

Le manioc est une plante domestiquée sur le continent américain avant d'atteindre les autres continents. En côte d'Ivoire il a été d'abord considéré comme une culture secondaire. C'est à partir de 1960 que le manioc attire l'attention des ivoiriens. Il devient une culture indispensable pour les ivoiriens. Il intervient dans la fabrication de plusieurs mets dont le plus prisé est l'attiéké. Les techniques de transformation de ses dérivés ont connu une évolution au fil des années. En 2000, le manioc ivoirien et ses dérivés alimentent deux marchés, le marché ouestafricain et le marché européen.

The cassava is a cultigen domesticated on the American continent, more precisely in the countries of South America. It was from this part that the manioc gained the African continent and in particular Ivory Coast. The cassava was introduced in Ivory Coast through the exchanges which the country had with Europeans. The first people which were in liaison with Europeans in Ivory Coast were obviously the coastal populations. This is why, the manioc developed in the zone lagoon before reaching the other areas of the country. At the beginning, the manioc had two characters, namely a character of basic food for the lagoons and a character of secondary culture for the populations of the center and North. The manioc was primarily regarded as a culture intended for the subsistence farming of the populations. But, beginning in the 1960s, the culture of the manioc became more significant to reach the peak two decades later. From this moment, the manioc changed character to become a culture of speculation as well as the cultures of revenues. The manioc then started to feed the domestic market of the Ivory Coast and even the markets of the West African inland areas. The manioc also feeds the European market in byproducts.

\section{INDEX}

Mots-clés : histoire des techniques, manioc, agriculture, savoir traditionnel, artisanat Keywords : history of technology, agriculture, cassava, traditional knowledge, craftsmanship Thèmes : Positions de thèse 


\section{AUTEUR}

\section{DAOUDA DIARRASSOUBA}

Originaire de la ville d'Issia dans la région du haut-Sassandra (centre-ouest de la Côte d'Ivoire), docteur en Histoire économique et des techniques et histoire économique, Daouda DIARRASSOUBA est actuellement enseignant du secondaire dans la ville de Tanda, région du Gontougo (Bondoukou) au Nord-Est de la Côte d'Ivoire. Il appartient à l'Université Alassane Ouattara de Bouaké. 